\title{
Risk Communication and Generic Preparedness: From Agent-based to Action-based Planning - A Conceptual Framework
}

\author{
P. Dickmann ${ }^{1^{*}}$, F. J. Apfel ${ }^{2}$ and R. Gottschalk ${ }^{3,4}$ \\ ${ }^{1}$ Dickmann Risk Communication (DRC), London, United Kingdom. \\ ${ }^{2}$ World Health Communication Associates (WHCA), Compton Bishop, United Kingdom. \\ ${ }^{3}$ Health Protection Authority, City of Frankfurt, Breite Gasse 28, 60313 Frankfurt, Germany. \\ ${ }^{4}$ Institute of Medical Virology, Hospital of the Goethe University of Frankfurt, Paul-Ehrlich-Str. 40, \\ 60596 Frankfurt, Germany.
}

\section{Authors' contributions}

This work was carried out in collaboration between all authors. Authors PD and RG have developed the original concept. Author PD has drafted the manuscript and authors FJA and RG have made substantial contributions to the concept and the manuscript. All authors have revised the manuscript critically and approved the final version.

Article Information

DOI: $10.9734 / B J M M R / 2016 / 24033$ Editor(s):

(1) Fuhong SU, ICU Laboratory, Erasme Hospital, Free University Brussels, Brussels, Belgium.

Reviewers:

(1) Marcos De Donato, Tecnológico de Monterrey, Mexico. (2) Diana C. Tapia-Pancardo, National Autonomous University of Mexico, Mexico. (3) Sukhmeet Minhas, Armed Forces Medical College, Pune, India. Complete Peer review History: http://sciencedomain.org/review-history/13293

Opinion Article

Received $31^{\text {st }}$ December 2015

Accepted $29^{\text {th }}$ January 2016

Published $14^{\text {th }}$ February 2016

\section{ABSTRACT}

Responses to recent infectious disease outbreaks, such as to Influenza Pandemic 2009 and the on-going Ebola outbreak in West Africa, reveal the need for new and strengthened approaches to risk communication and governance. The article argues for a fundamental re-conceptualisation of current approaches to risk communication, preparedness planning and response. It calls for a reframing of the way we currently identify and respond to outbreaks around a set of core behaviour-based response patterns. This new model moves away from the current risk communication focus on a plethora of agent-specific threats to five generic response patterns that are based on socially relevant response activities such as 1) controlling vectors, 2) enhancing 
hygiene, 3) isolation of the sick, 4) protection of the well, and 5) systemic protection of people and their environments. Emphasis is placed on gaining relevant insights into the context specific needs of different communities related to these five patterns. Governance structures are then built and evaluated based on their capacity to collect, communicate, share and prepare the public to take appropriate action related to the five different patterns before, during and after an event. Reframing risk communication and preparedness approaches around a better understanding of the determinants of these general behavioural patterns in infectious control could strengthen infection control literacy, response competence and build resilience of both individuals and health systems to address future epidemics, pandemics and other public health threats.

Keywords: Governance; risk communication; generic preparedness; pandemic preparedness; influenza pandemic; public health.

\section{SIGNIFICANCE TO PUBLIC HEALTH}

The current rationale of risk communication and preparedness planning builds on a plethora of agent specific threats. This requires continuous expert assessment and recommendations and thus increases the dependence on experts and reinforces the current dominance of the biomedical paradigm.

This article describes a new social action model that is built on five generic response patterns. These "patterns" describe specific socially relevant individual behaviour and public health system activities that can guide risk communication approaches before, during and after an event. The benefits of applying this new risk communication approach would be to improve the infection control literacy and competence of both individuals and public health systems to address and support socially relevant actions in each of the five areas. Developing such competencies could reduce dependence on experts, shift biomedical paradigm to more socially relevant approaches and help build and strengthen peoples' and systems' resilience in addressing future epidemics, pandemics and other public health threats.

\section{BACKGROUND}

Recent infectious disease outbreaks, such as the Influenza Pandemic 2009 ("influenza A [H1N1]pdm09") and the Ebola outbreak in West Africa highlight the fact that current risk communication, governance and structural approaches to preparedness and response planning for infectious disease outbreaks are not sufficient to prepare for or adequately engage with the public and various stakeholders (e.g. travel, trade, international organizations, etc.). This has had a significantly negative impact on the speed, effectiveness and efficiency of response measures [1]. The knowledge and perception gap between what public health officials consider important and what the key drivers of affected communities actually are, was wide during Influenza pandemic and is even wider (and almost disconnected) during the Ebola outbreak response [2-4].

National and international preparedness planners have started examining how underlying systems and structures are contributing to response failures. Problems related to one-directional communication, lack of trust and relationship building between providers and people and between relevant sectors and stakeholders are being identified as higher priority issues needing attention. Experts are calling for better engagement strategies with communities, the need for better "listening", on-going involvement and more culturally sensitive action oriented recommendations [5].

While these change suggestions are most definitely a move in the right direction we believe that implementing them requires new governance and communication approaches. We argue for a fundamental re-conceptualisation of preparedness planning that builds on more systematic, collaborative and smarter approaches to governance for health and a reframing of how we communicate infectious threats with the public [6].

In this article we particularly focus on how communication could be reframed from its current fixation on multiple agent-based response plans to a generic social action-based preparedness planning paradigm. We identify five infection control patterns that people can adopt to protect themselves and others. These patterns are determined by socially relevant action people can take and not solely based on conventional scientific distinctions into virus, bacteria or parasites nor on the epidemiological 
rational of transmission patterns (vector-borne diseases, direct contact, etc.). The key question addressed in each of the patterns in our approach is what actions people can take to protect themselves, their families and communities? The risk communication challenge focuses on gaining relevant insights into the context specific needs of different communities related to these five patterns. Governance structures are then built and evaluated based on their capacity to collect, communicate, share and prepare the public to take appropriate action related to the five different patterns before, during and after an event.

\subsection{Hypothesis}

It is our hypothesis that risk communication governance systems that adopt a socially relevant pattern of infection control approach will enable people to become more independent and informed participants and thereby lead to earlier, faster, smoother and smarter responses [7].

\section{INTRODUCTION OF A GENERIC APPROACH: FROM AGENT-BASED PLANNING TO AN ACTION-BASED GENERIC PREPAREDNESS}

To this end we propose to modify the conceptual framing currently surrounding public health infectious disease risk communication governance. We propose that - rather than a directive agent-based approach - an actionbased approach should be adopted in preparedness planning and response. Our new approach is not based on single causative agents, but on pragmatic, socially relevant patterns of infection control. We argue for a more generic action oriented preparedness planning model.

\subsection{Socially Relevant Patterns of Infection Control - A Framework}

\subsubsection{Patterns of infection control}

We suggest different patterns based on socially relevant infection control behaviours related to actual mode of transmission and needed epidemiologically based preventive actions. We first divide diseases between those that are not or only rarely transmissible between humans and those that are human-to-human transmissible. We then suggest five behavioural patterns related to the key threat element (e.g. a known vector) and the core social prevention action required (e.g. vector control).

\subsubsection{Five socially defined patterns}

\subsubsection{Not (1.) or rarely (2.) transmissible between humans}

1. Control vectors: Some diseases, such as Dengue fever, Malaria or Chikungunya fever, are transmissible via animate vectors - a direct transmission from human to human does not take place. Infection control measures are based on the controlling and avoidance of vector transmission, e.g. using insecticides, repellents, disinfection, decreasing room temperature, draining reservoirs, etc.

Pattern objective: Prevent transmission by a known vector

Pattern action: Control vector

2. Enhance hygiene: Some diseases, such as Cholera or Salmonellosis, are transmissible via indirect contact only (contaminated water or food, Bush Meat, handles, clothes etc.). Hands/body fluids are direct possibilities of transmission and a transmission from human to human is extremely rare, if ever. The governing principle of infection control here is the prevention of indirect (and direct) transmission, e.g. provision of clean water, cleaning of contact surfaces, increased hygiene including avoidance of handshaking etc.

Pattern objective: Prevent transmission of a known source

Pattern action: Enhance hygiene

\subsubsection{Transmissible between humans (3., 4., 5.)}

3. Isolation of sick: A large variety of diseases are transmissible from a sick person to other humans via direct contact. Ebola virus is one example. The governing principle of infection control is to avoid contact with a sick person.

Pattern objective: Prevent contact with sick humans

Pattern action: Isolation of sick

4. Prevent exposure to sick humans: Some diseases are very easily 
transmissible. Exposure to a sick person can facilitate an infection. The Severe Acute Respiratory Syndrome (SARS) and the Middle East Respiratory Syndrome (MERS) are examples of diseases that are transmissible from a sick person to another human via droplets and aerosols but only after the onset of typical signs of the disease. Infection control actions here aim at detecting early signs and symptoms and prevent the airborne transmission, e.g. increased hygiene including masks and gloves, etc.

Pattern objective: Prevent exposure to sick humans

Pattern action: Protect the well

5. Prevent exposure: Some diseases are transmissible from human to human via droplets and aerosols before the onset of, or without, symptoms. Influenza and Measles are examples. The infection control approach here is likely to be highly sophisticated and targeted at the avoidance of air-borne transmission and contact, e.g. increasing levels of general hygiene, masks, gloves, interpersonal spatial distancing (not social distancing).

Pattern objective: Prevent exposure

Pattern action: Systemic protection of people and their environments.

\section{DISCUSSION}

Reframing risk communication approaches around these five social behavioural patterns allows for a simpler, more accessible framework for action. It requires, however, an understanding of the social, cultural, religious customs and belief systems contexts within which needed change in the different behaviour patterns might be sought. Without such understanding risk communications will falter. During the Ebola outbreak in West Africa, for instance, people did not adopt the recommendation to avoid touching their sick relatives as such behaviours go against traditional community care, burial practices and religious beliefs. Changing such traditional patterns requires a new approach to risk communication. Such changes are only realisable through better and more collaborative governance and risk communication systems that can find a common ground to develop solutions that work for both sides: the infection control side - and the people side.

\section{LIMITATIONS AND STRENGTH}

Utilising these five relevant patterns provide a framework and platform for identifying and addressing the key principles from the infection control side (e.g. prevent transmission by a known vector) and the people side (e.g. vector control). The exact changes required will depend on the different settings and societies and need to be developed with people and their public health services. Such preparedness planning requires more than preparing and delivering health messages: it requires governance and risk communication systems that understand the social dimensions and have taken steps to build on-going relationships with affected communities so as to be able to explore pragmatic and realistic options. A key advantage of reducing risk communication to five relevant patterns is that it can empower communities with a new independence from experts. Risk communication focussed on single diseases and expert opinions providing scientific information about the particular causative agent is too often one-directional. These five social action related patterns of infection control demand dialogue and represent a new conceptual approach that facilitates the development of context specific collaborative and enabling risk communication for outbreaks with public health relevance.

\section{CONCLUSION}

Reframing how we conceptualise generic preparedness around an agreed set of behavioural patterns in infectioun control has the potential to improve the preparedness planning for public health emergencies and may help build and strengthen resilience of the general public to address future epidemics, pandemics and other public health threats. Enhancing capacities to support needed behaviour changes through the use of collaborative risk communication approaches can also help strengthen public health systems on all levels of governance, from the local to global.

\section{CONSENT}

It is not applicable.

\section{ETHICAL APPROVAL}

It is not applicable. 


\section{ACKNOWLEDGEMENTS}

The authors have not received financial support or intellectual services for the development of the conceptual framework.

\section{COMPETING INTERESTS}

Authors have declared that no competing interests exist.

\section{REFERENCES}

1. Fineberg HV. Pandemic preparedness and response--lessons from the $\mathrm{H} 1 \mathrm{~N} 1$ influenza of 2009. NEJM. 2014; 370(14):1335-42.

2. Wolz A. Face to face with ebola - An emergency care center in Sierra Leone. NEJM. 2014;371(12):1081-3.

3. Wiwanitkit V. Ebola, fear and preparedness. Epidemiol Health. 2014; 36:e2014015.
4. Ryschon TW. Ebola control measures and inadequate responses. Lancet. 2014;pii:S0140-6736(14):61346-55.

5. Guerrier G, D'Ortenzio E. Africa. Ebola virus control needs local buy-in. Nature. 2014;513(7518):315.

6. Kickbusch I, Gleicher D: Governance for health in the 21st century: A study conducted for the WHO Regional Office for Europe; 2011.

Available:http://www.euro.who.int/ data/a ssets/pdf file/0010/148951/RC61 InfDoc6. $\underline{\text { pdf }}$

(Accessed 07 March 2015)

7. Dickmann $P$, McClelland $A$, Gamhewage G, Portela de Souza P, Apfel F. Making sense of communication interventions in public health emergencies - an evaluation framework for risk communication. Journal of Communication in Healthcare. 2015;8(3):233-240.

(c) 2016 Dickmann et al.; This is an Open Access article distributed under the terms of the Creative Commons Attribution License (http://creativecommons.org/licenses/by/4.0), which permits unrestricted use, distribution, and reproduction in any medium, provided the original work is properly cited.

Peer-review history:

The peer review history for this paper can be accessed here: http://sciencedomain.org/review-history/13293 